\title{
Are We Ready? The Construct of Subjective Cognitive Impairment and its Utilization in Clinical Practice: A Preliminary UK-Based Service Evaluation
}

\author{
Amy Jenkinsa, ${ }^{*}$, Andrea Talesa, Jeremy Treea and Antony Bayerb \\ aDepartment of Psychology, Swansea University, Wales, UK \\ bSchool of Medicine, Cardiff University, Wales, UK \\ Accepted 20 August 2015
}

\begin{abstract}
Extensive research on the concept of mild cognitive impairment (MCI) as a potential prodromal stage of dementia has highlighted the likelihood that abnormalities in information processing occur at even earlier stages in the disease process with research increasingly focused on the relatively new concept of subjective cognitive impairment (SCI). An individual with SCI will experience cognitive impairment solely on a subjective level, which is in contrast to an individual with MCI who will also experience cognitive impairment at an objective level. SCI is believed to be a risk factor for development of MCI. This qualitative service evaluation aimed to determine how much is known about SCI and how it is currently managed in specialist clinical practice in the UK. An email-based questionnaire containing a vignette of an individual presenting with SCI was distributed to 112 memory clinics requesting information on their most likely approach to such an individual. The $21 \%$ response rate evinces potential time pressure within clinical services that may preclude research participation and/or a lack of issue salience at present. However, the data from those who responded provide an important insight into 'where we are now' in relation to this issue. Analysis revealed main themes associated with SCI, namely the factors that influence what action is taken when an individual presents and what further investigations are performed, the multiplicity of potential outcomes experienced, and the barriers clinicians may face. The findings highlight the need for a coherent and consistent framework in relation to the management of SCI.
\end{abstract}

Keywords: Dementia, health services research, memory clinic, mild cognitive impairment, subjective cognitive impairment

*Correspondence to: Amy Jenkins, College of Human and Health Sciences, Department of Psychology, Room 809, Vivian Building, Swansea University, Singleton Park, Swansea, SA2 8PP, UK. Tel.: +44 01792 604219; Fax: +44 01792 295679; E-mail: 643775@swansea.ac.uk.

\section{INTRODUCTION}

Mild cognitive impairment (MCI) is a well-documented and clinically recognized potential prodromal stage of dementia $[1,2]$. Subjective cognitive impairment (SCI), in which subjective changes in memory and cognition are reported in the absence of objective evidence of abnormality [3], has, however, received comparatively little attention. Although SCI is etiologically heterogeneous, individuals with this disorder are significantly more likely to decline to MCI or dementia diagnosis compared to demographically matched healthy adults free of SCI (hazard ratio 4.5; 95\% CI 1.9-10.3), indicating that SCI often represents a harbinger of further decline [4]. Nevertheless, research and clinical practice in relation to prodromal dementia have tended to focus on MCI, despite indications that approximately $10 \%$ of adults aged 65 and above in the UK have MCI and $42 \%$ have self-reported memory complaints [5].

Irrespective of the increased risk for dementia, the assumption that SCI is benign and not associated with problems of significance is increasingly challenged. Irrespective of whether SCI represents an earlier prodromal stage of dementia or not, some individuals will suffer emotional distress because their concerns are not validated and they live with fear of significant underlying brain pathology and impending dementia. Concurrent mood disturbance and other reversible conditions may be overlooked and effective treatment denied. Quality of life may be significantly affected and perceived workplace performance impaired [6], social activities restricted, and driving avoided [7]. Furthermore, by the time a person with SCI who is on the Alzheimer's disease (AD) pathway develops MCI, irreversible neuronal damage will have occurred $[1,8-10]$ and the potential benefit of disease-modifying treatment may already be reduced. Such research evidence suggests that effective intervention to delay or prevent decline may likely best be targeted at the earliest symptomatic stage of SCI, in which cognitive functioning is still relatively preserved. 
In parallel with this research-based evidence, the growing awareness of AD and associated disorders among non-specialist clinicians and the general public is leading to increasing referrals of individuals who report changes in memory and cognition to specialist services [11-13]. In a recent report from a memory clinic in Stockholm, 38\% of patients were classified as having SCI [14]. Similarly, a recent survey of neurology-led UK memory clinics in 2014 suggested a mean of $27 \%$ of attendees had 'benign memory complaints' and the percentage has increased significantly over recent years. However, neurology-led clinics see predominantly younger patients and most memory assessment services in the UK are run by old age psychiatrists and geriatricians so further information about a wider range of memory services is required. The aim of this study is to determine levels of awareness about SCI as a construct in more typical specialist clinical practice in England and Wales (i.e., by memory clinics/services) and what clinicians do when faced with individuals meeting the criteria for SCI classification [3].

\section{MATERIALS AND METHODS}

For this cross-sectional, service evaluation study, an email was distributed to every Royal College of Psychiatrists accredited memory clinic in England and to all the memory services in Wales. Within this email was a vignette (Table 1) describing a fictitious individual displaying typical signs of SCI. This vignette was accompanied by an open question asking what the memory service would do in this situation and closed questions relating to potential management options. Prior to this study, three memory clinic clinicians assessed this evaluation tool in order to validate the appropriateness of the questions and the accompanying vignette. The vignette was relatively sparse in order to encourage clinic-specific and a wide-range of responses.

As replies could not be anonymized, all identifiable information from the email responses was removed before analysis. Thematic analysis was employed for the free text responses to the open question, which was realist driven, inductive, and bottom-up [16]. The responses were read and re-read by the principal investigator (AJ) who made initial comments and codes. The process was repeated twice more until individual codes were identified, and then grouped into themes in order to highlight commonalities among them. The code and theme development were subsequently checked by another member of the research team (AT).

\section{RESULTS}

Of 112 questionnaires sent out, 23 responses were received, representing a $21 \%$ response rate. Table 2 details responses to the first question about how teams would respond to patients with SCI. The identified themes and their relevant sub-themes are listed in descending order of response frequency.

\section{Theme One: 'Action is dependent on'}

This theme represents what the memory teams said would influence the actions they would take in managing someone like the 'Mr X' described in the vignette. Four sub-themes emerged, namely action would depend upon the patient's premorbid ability and educational background, their activities of daily living (ADL), the presence of functional changes, and whether discrepancies between ADLs, functionality, and cognitive difficulties were identified.

\section{Theme Two: 'Further investigations'}

This theme represents what further investigations the memory teams would carry out if they were managing the case of ' $\mathrm{Mr} \mathrm{X}$ '. Seven sub-themes emerged: namely (1) the team would ensure an informant interview was carried out; (2) they would screen for reversible causes of SCI; (3) some clinicians reported that they would refer ' $\mathrm{Mr} \mathrm{X}$ ' for more comprehensive neuropsychological assessment; (4) they would refer ' $\mathrm{Mr} \mathrm{X}$ ' for a more comprehensive mental health assessment; (5) referral for further scanning; (6) referral to occupational therapy; and (7) requests for a patient to have a driving assessment.

\section{Theme Three: 'Outcomes'}

This theme is represented by six sub-themes and is related to the various outcomes patients experience, namely (1) most memory teams reported that they would reassure and discharge the individual if, after further investigation, no problems were identified; (2) many would reassure then discharge the individual and ask the GP to monitor and re-refer if problems persist or worsen; (3) they would arrange a follow up appointment; (4) the team would reassure, discharge, and signpost for additional support; (5) they would employ the strategy of 'watchful waiting'; and (6) some reported that they would not have initially even accepted the referral. 
Table 1 Vignette and questions distributed to memory clinics by email

$\mathrm{Mr} \mathrm{X}$ was referred to your clinic because he has noticed that he has developed some cognitive difficulties. He has no history of mental health problems, drug or alcohol misuse, and no history of vascular problems. He scored 29/30 on the MMSE, and 98/100 on the ACE-R.

*Your responses will be confidential and anonymised*

(1) As a team what would you do in this situation?

(2) Please select which of the below applies to you and your team (you can select more than one)

- generally reassure and discharge yes/no

- do not discharge, but employ an open door principle yes/no

- diagnose them with subjective cognitive impairment yes/no

- routinely refer for detailed neuropsychological testing yes/no

- routinely refer for a mental health assessment yes/no

- routinely refer for neuroimaging yes/no

- arrange future follow up for repeat assessment yes/no

- other 
Table 2 Themes and sub-themes identified in response to question asking

\begin{tabular}{|c|c|}
\hline Theme & Sub-themes \\
\hline $\begin{array}{l}\text { 1. Action is } \\
\text { dependent on... }\end{array}$ & $\begin{array}{ll}\text { - } & \text { Premorbid ability and educational background } \\
\text { - } & \text { Activities of daily living (ADL) } \\
\text { - } & \text { Functional changes } \\
\text { - } & \text { Discrepancies between ADL/functionality and cognitive } \\
& \text { difficulties }\end{array}$ \\
\hline $\begin{array}{l}\text { 2.Further } \\
\text { investigations }\end{array}$ & $\begin{array}{ll}\text { - } & \text { Informant interview } \\
\text { - } & \text { Screen for reversible causes of SCI } \\
\text { - } & \text { Neuropsychology assessment } \\
\text { - } & \text { Fuller mental health assessment } \\
\text { - } & \text { Further scanning } \\
\text { - } & \text { Refer to occupational therapy } \\
\text { - } & \text { Driving assessment }\end{array}$ \\
\hline 3.Outcomes & 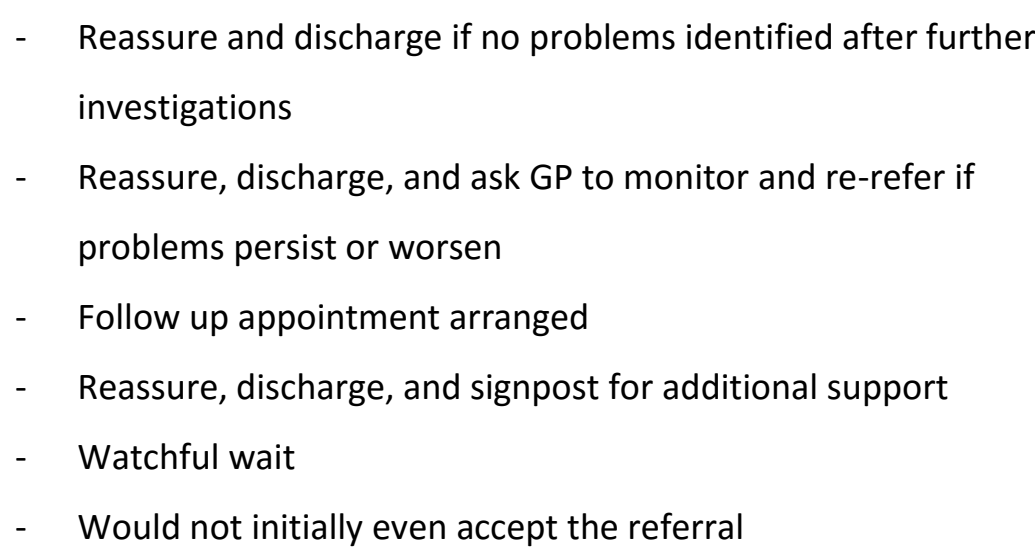 \\
\hline 4.Barriers & $\begin{array}{ll}\text { - } & \text { Resources available } \\
\text { - } & \text { Shared care protocol } \\
\text { - } & \text { Commissioning regulations } \\
\text { - } & \text { Wanting to avoid making patient anxious }\end{array}$ \\
\hline
\end{tabular}

\section{Theme Four: 'Barriers'}

This is related to the difficulties teams face when managing their patients. Four sub-themes represent this theme. The first barrier identified was related to regarding the resources available to the team, e.g., staff numbers. Second, there are difficulties when some of the clinics have a shared protocol with another governing body, and 
third when they have shared commissioning regulations. Lastly, some reported that avoiding making the patient anxious would be a barrier with respect to case management.

Table 3 shows the responses to the closed questions that the memory team selected regarding the management of the fictitious case of ' $\mathrm{Mr} \mathrm{X}$ '. Five of the 23 clinics that responded did not complete any questions in this section. A patient being 'gently reassured and discharged' was the most frequently selected from the list at $67 \%$ (12 out of 18 clinics). A patient being 'routinely referred for more detailed neuropsychological testing' and also 'routinely referred for neuroimaging' were both selected by $44 \%$ of the respondents ( 8 out of 18 clinics). A 'routine referral for a mental health assessment' was selected by $24 \%$ of the respondents (4 out of 17 clinics), and $22 \%$ would 'arrange a future follow up for a repeat assessment' (4 out of 18 clinics). An open door policy would be in place (without being discharged) by $18 \%$ of the respondents ( 3 out of 17 clinics), and only $6 \%$ said that they would diagnose SCI (1 out of 17 clinics).

Table 3 Responses to question concerning actions subsequent to the diagnosis of SCI

Number (\%) of positive

responses

Gently reassure \& discharge

$12(67)$

Do not discharge but employ an open

door policy*

Diagnose $\mathrm{SCl}^{*}$

$1(6)$

Routinely refer for more detailed

$8(44)$

neuropsychological testing

Routinely refer for mental health

assessment*

Routinely refer for neuroimaging

8 (44)

Arrange future follow up for repeat

$4(22)$

assessment

* $n=17$

\section{DISCUSSION}

The aim of this service evaluation was to explore the actions that memory clinics in England and Wales would take at the present time in response to the presentation of an individual with subjective memory or cognitive changes commensurate with the construct of SCI. 
It is clear that a relatively small proportion of responses were received and thus the outcome of this service evaluation should be interpreted accordingly, in that their responses may not be representative of all memory services in the UK. The reasons for this response rate are not known but to speculate it is possible that it represents a lack of 'issue salience' [17]. Thus the issue of SCI may not be deemed as an important one among clinical teams at present, despite this being an area of growing research importance and of concern to patients. Alternatively, response was simply precluded due to workload pressure and greater priorities.

It is, however, also clear that the actions of the clinics that responded were highly variable. For instance, very little consensus was evident regarding the most likely outcome for SCI patients across different clinics. For example, our survey revealed that while some would carry out further investigations and then reassure and discharge if no problems were identified, others would routinely arrange a follow-up appointment, while some would not even have accepted such a referral in the first place. One can argue therefore that such heterogeneity in response urgently needs addressing in order to ensure continuity of care across the National Health Service. Neither MCI nor SCI are within the Diagnostic and Statistical Manual 5 (DSM-5) or the International Classification of Diseases 10 (ICD-10). This is likely to contribute to the lack of clarity and understanding about these diagnostic categories.

Furthermore, the action taken by a clinic was also mediated by presenting features not mentioned in the vignette, but predictable all the same, namely premorbid ability and educational background, and whether or not they had experienced changes in their ADL or functional changes, i.e., a similar strategy to that applied to MCI. Additional assessments were also reported. Most frequently mentioned was the requirement for an informant interview, with some reporting that they would screen for potentially reversible causes of SCI not covered in the vignette. Due to a lack of clarity regarding what was causing 'Mr X's' cognitive difficulties, a number of clinics said that they would seek additional information using neuropsychological assessment and further neuroimaging, whereas others reported that they would routinely perform such levels of assessment. Despite the vignette stating that ' $\mathrm{Mr} \mathrm{X}$ ' had no history of mental health problems, only some of the clinics said that they would refer the patient for a more comprehensive mental health assessment. Least frequently mentioned were referrals to occupational therapy and driving assessments. It is clear, therefore, that little consistency regarding the outcomes for people with SCI occurs in practice.

Finally, a number of difficulties were identified which may act as barriers to the management of SCI. Fundamentally the main difficulty for clinics is the lack of resources, specifically staff numbers and available hours to take on a greater workload. Some clinics had difficulties associated with shared protocols and commissioning regulations, and others reported that they were concerned that investigations may invoke unnecessary anxiety in individuals reporting SCI if they were not showing signs of objective impairment. It is clear from these findings that the way SCI is managed across various memory clinics is heterogeneous. Lack of coherence is not generally considered to be best clinical practice, and is therefore indicative of urgent attention. For instance, a patient showing signs of SCI in one area of the UK may be referred to their local memory team, receive a full investigation with follow up appointments. Elsewhere, a similar patient referral will not even be accepted by the memory team, in which case it is possible that potentially reversible etiology will not be investigated further. Thus there appears to be a need for national guidance on the response to individuals presenting with SCI.

From those who responded, the identification of themes and sub-themes suggests three key messages for all those who are likely to be involved in the management of someone with cognitive difficulties.

\section{Thorough investigation}

Five respondents stated that they would need further information prior to making any decisions regarding best course of action, with a minority reporting that they would carry out additional investigations and explore the patient's concerns further, with additional neuropsychological testing and/or neuroimaging (8 out of 18 respondents). Emphasis was placed on seeking further information from an informant, such as a close family member. The many potential factors associated with SCI $[15,18]$ justify a thorough specialist assessment in a memory clinic, with the aim of identifying what the problem is, its etiology, whether it can be treated, and how it can be managed. Ten out of the 18 respondents said they would not do additional investigations such as neuropsychological testing or neuroimaging. Only a minority (4 out of 18 respondents) of services would follow up a patient with SCI, but this may be due to limited resources and the need to prioritize activity by focusing on those with greatest cognitive and functional deficits.

\section{Using the term SCI}

Only one service out of 17 currently make a diagnosis of SCI or attempt to diagnose individuals who complain of changes in memory/cognition, but it is unclear if this is due to lack of familiarity with the term, or a 
reluctance to endorse a patient's subjective complaints with a diagnostic label. Certainly emotional distress related to cognitive symptoms may be minimized if concerns are validated and not disregarded.

Given the growing interest in SCI as an early presentation of impending dementia, use of the term for diagnosis will help to promote necessary research and may have future personal and societal benefits if effective preventive strategies are developed.

Table 4 Best practice recommendations for people presenting with SCI

1. Premorbid ability and educational background must be taken into account

2. Activities of daily living (ADL) and functional changes should be assessed

3. Discrepancies between ADL/functionality and cognitive difficulties should be a cause for concern and investigated further

4. Further investigations such as detailed neuropsychological testing, neuroimaging and/or mental health assessment should be carried out if required

5. An informant interview should be an essential feature of the assessment process

6. All possible reversible causes of $\mathrm{SCl}$ should be assessed and treated if identified

7. Follow-up appointment should be offered to assess changes, or an open door policy with easy access should be implemented if patient, family or general practitioner have any new concerns in the future

\section{Consistent approach to assessment and management is desirable}

There was no agreement on the appropriateness of further investigations or follow up of people with SCI. A lack of consistency is generally not regarded as best practice and best practice recommendations would seem appropriate. Based on the study responses, our provisional best practice recommendations are presented in Table 4.

To conclude, we aimed to explore what strategies were in place in memory services in the UK for someone displaying signs of SCI. The findings highlight a rather urgent need for a coherent and consistent framework to be introduced for the management of SCI. Three central messages have been put forward based on the themes and sub-themes identified. What is also clear is that several years ago the concept of MCI was the topic of similar debate to that surrounding SCI today and that MCI is now a widely recognized clinical diagnosis. As SCI may represent an increased risk factor for both MCI and dementia we argue that its clinical investigation and management be addressed more fully and a timely consensus reached.

\section{ACKNOWLEDGMENTS}

This service evaluation was carried out in accord with the ethical standards of the Swansea University Department of Psychology ethics committee. Authors' disclosures available online (http://j-alz.com/manuscriptdisclosures/15-0541r1). 


\section{REFERENCES}

[1] Jack CR Jr, Knopman DS, Jagust WJ, Petersen RC, Weiner MW, Aisen PS, Shaw LM, Vemuri P,Wiste HJ,Weigand SD, Lesnick TG, Pankratz VS, Donohue MC, Trojanowski JQ (2013) Tracking pathophysiological processes in Alzheimer's disease: An updated hypothetical model of dynamic biomarkers. Lancet Neurol 12, 207-216.

[2] Morris JC, Price JL (2001) Pathologic correlates of nondemented aging, mild cognitive impairment, and early-stage alzheimer's disease. J Mol Neurosci 17, 101-118.

[3] Stewart R (2012) Subjective cognitive impairment. Curr Opin Psychiatry 25, 445-450.

[4] ReisbergB, ShulmanMB,Torossian C, Leng L, ZhuW(2010) Outcome over seven years of healthy adults with and without subjective cognitive impairment. Alzheimers Dement 6, 11-24.

[5] Stephan BC, Matthews FE, McKeith IG, Bond J, Brayne C (2007) Medical Research Council Cognitive Function and Aging Study. Early cognitive change in the general population: How do different definitions work? J Am Geriatr Soc 55, 1534-1540.

[6] McIntyre RS, Soczynska JZ, Woldeyohannes HO, Alsuwaidan MT, Cha DS, Carvalho AF, Jerrell JM, Dale RM, Gallaugher LA, Muzina DJ, Kennedy SH (2015) The impact of cognitive impairment on perceived workforce performance: Results from the international mood disorders collaborative project. Compr Psychiatry 56, 279-282.

[7] Desai AK, Schwarz L (2011) Subjective cognitive impairment: When to be concerned about 'senior moments'. Curr Psychiatry 10, 3145.

[8] Sperling RA, Jack CR Jr, Aisen PS (2011) Testing the right target and right drug at the right stage. Sci Trans/ Med 3, $111 \mathrm{~cm} 33$. [9] Aizenstein HJ, Nebes RD, Saxton JA, Price JC, Mathis CA, Tsopelas ND, Ziolko SK, James JA, Snitz BE, Houck PR, Bi W, Cohen AD, Lopresti BJ, DeKosky ST, Halligan EM, Klunk WE (2008) Frequent amyloid deposition without significant cognitive impairment among the elderly. Arch Neurol 65, 1509-1517.

[10] Kryscio RJ, Abner EL, Cooper GE, Fardo DW, Jicha GA, Nelson PT, Smith CD, Van Eldik LJ, Wan L, Schmitt FA (2014) Self reported memory complaints: Implications from a longitudinal cohort with autopsies. Neurology 83, 1359-1365.

[11] Wang B, Guo QH, Chen MR, Zhao QH, Zhou Y, Hong Z (2011) The clinical characteristics of 2,789 consecutive patients in a memory clinic in China. J Clin Neurosci 18, 1473-1477.

[12] Bayer AJ (2012) Memory Clinics. In Pathy's Principles and Practice of Geriatric Medicine Fifth Edition Sinclair A, Morley J, Vellas B, eds. John Wiley, Chichester, pp. 1061-1071.

[13] Lehrner J, Moser D, Klug S, Gleiß A, Auff E, Dal-Bianco P, Pusswald G (2014) Subjective memory complaints, depressive symptoms and cognition in patients attending a memory outpatient clinic. Int Psychogeriatr 26, 463-473.

[14] Garcia-Ptacek S, Cavallin L, K ${ }^{\circ}$ areholt I, Kramberger MG, Winblad B, JelicV, EriksdotterM(2014) Subjective cognitive impairment subjects in our clinical practice. Dement Geriatr Cogn Disord Extra 4, 419-430.

[15] Jessen F, Amariglio RE, van Boxtel M, Breteler M, Ceccaldif M, Ch'etelat G, Dubois B, Dufouil C, Ellis CA, van der FlieR, Glodzik WM, van Harten L, de Leon AC, McHugh MJ, Mielke P, Molinuevo MM, Mosconi JL, Osorio L, Perrotin RS, Petersen A, Rabin RC, Rami LA, Reisberg L, Rentz B, Sachdev DM, de la Sayette PS, Saykin V, Scheltens AJ, Shulman P, Slavin MB, Sperling MJ, Stewart RA, Uspenskaya R, Vellas O, Visser B, Wagner PJ, Subjective Cognitive Decline Initiative (SCD-I) Working M, Group (2014) A conceptual framework for research on subjective cognitive decline in preclinical alzheimer's disease. Alzheimers Dement 10, 844-852.

[16] BraunV, ClarkeV (2006) Using thematic analysis in psychology. Qual Res Psychol 3, 77-101.

[17] Sheehan KB, McMillan SJ (1999) Response variation in e-mail surveys: An exploration. J Advert Res 39, 45-54.

[18] Goshorn RK (1998) Chronic fatigue syndrome: A review for clinicians. Seminars Neurol 18, $237-242$. 